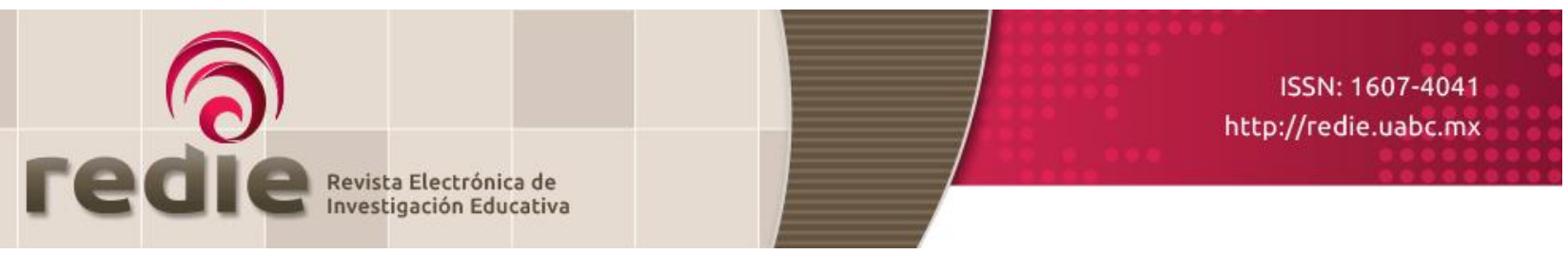

Vol. 21, 2019/e34

\title{
Estudio sobre la creatividad relacionada con la habilidad de programadores universitarios
}

\section{Study on Creativity Related to the Ability of University Programmers}

\author{
Claudia Islas Torres (1) cislas@cualtos.udg.mx \\ María del Rocío Carranza Alcántar (1) mcarranza@cualtos.udg.mx \\ Antoni Pérez Poch (2) a.perezpoch@gmail.com \\ Nuría Salán Ballesteros (2) nuria.salan@upc.edu \\ (1) Universidad de Guadalajara \\ (2) Universidad Politécnica de Cataluña \\ (Recibido: 25 de agosto de 2017; Aceptado para su publicación: 23 de noviembre de 2017)
}

Cómo citar: Islas, C., Carranza, M. del R., Pérez, A. y Salán, N. (2019). Estudio sobre la creatividad relacionada con la habilidad de programadores universitarios. Revista Electrónica de Investigación Educativa, 21, e34, 1-12. doi:10.24320/redie.2019.21.e34.2143

\section{Resumen}

La habilidad de programar es una de las capacidades que desarrollan o, en su caso, potencializan los estudiantes de Ingeniería en Computación, algunos estudios en la literatura parten de la premisa de que dicha habilidad tiene asociación directa con la capacidad creativa de los estudiantes; a partir de lo anterior, la presente investigación tuvo como objetivo identificar la relación que existe entre la creatividad de un estudiante de Ingeniería en Computación y su rendimiento como programador, representado por el promedio obtenido en ciertas asignaturas. El método aplicado fue cuantitativo, transversal, de alcance explicativo y para la recuperación de datos se utilizó el test CREA. Los resultados indican que la creatividad no es incidente para la habilidad de programar y que son otros factores los que influyen para desarrollarla, tales como la motivación incentivada principalmente por el docente y el contexto que rodea al programador.

Palabras clave: Creatividad, Programación, ingeniería de sistemas, ciencias computacionales.

\section{Abstract}

The ability to program is one of the capacities developed or, if applicable, computer engineering students, some studies in the literature start from the premise that this ability has a direct association with the creative capacity of students; From the above, the research reported was presented had the objective of identifying the relationship between the creativity of an engineering student in computing and his performance as a programmer, represented by the average obtained in certain subjects. The applied method was quantitative, transversal, of explanatory scope and for the recovery of data, the CREA test was used. The results indicate that creativity is not an incident to the ability to program and that other factors influence the development, such as the motivation encourage mainly by the teacher and the context surrounding the programmer. 


\section{Introducción}

La revolución y evolución acelerada de hardware y software requiere de personas que tengan la capacidad de desarrollar pensamiento divergente y razonado (González, Estrada y Martínez, 2016) que les lleve a plantear soluciones o propuestas ante la diversidad de situaciones sensibles de resolverse a través de algoritmos computables.

Por su parte, Yates (1999) señala que programar es quizá el corazón de las Ciencias de la Computación, además de que puede considerarse más un arte que una ciencia o una ingeniería, de tal manera que se ha constituido en una opción importante para la resolución de problemas, por lo que se requiere no sólo de analizar o implementar sino de llevar a cabo todo el proceso de manera general y completa, lo que conlleva que se desarrollen competencias que permitan utilizar la creatividad.

En la actualidad diversos programas se utilizan para facilitar no sólo la comunicación sino la vida diaria, muchos de ellos son manipulados por niños o jóvenes sin tener conciencia del potencial que éstos tienen para desarrollar su creatividad. Con ello puede argumentarse la importancia que tiene programar, pues se requiere de capacidades para diseñar soluciones a problemas para luego convertirlas en programas.

En este sentido, enseñar y aprender a programar pareciera ser una tarea compleja, puesto que requiere de habilidades cognitivas estructuradas y lógicas que lleven al docente a enseñar lo que el estudiante debe comprender de la programación, en términos de su estructura, sentido, organización, aplicabilidad, abstracción, etc., por lo que programar implica desarrollar capacidades que ayuden a los estudiantes a convertirse en pensadores creativos, analíticos y prácticos (Yates, 1999).

Aunado a lo anterior, es importante resaltar que los docentes encargados de guiar el aprendizaje de los programadores debieran fomentar en ellos el desarrollo de la creatividad a través de temáticas como los videojuegos, cómic digital, producción de videos, robótica y brigadas tecnológicas, que si bien no son parte del plan curricular deben considerarse como apoyo en las enseñanzas para reforzar habilidades tales como pensamiento crítico, creatividad y trabajo colaborativo (Escobar, 2015), ya que no tiene sentido aprender a programar sólo a partir de memorizar las instrucciones estructuradas ni planteando ejemplos para cada una de ellas, sino que se requiere desarrollar otras habilidades que les permitan programar de manera más estructurada el trabajo requerido (Espeso, 2015).

A partir de las premisas anteriores, el presente estudio concentra los resultados de una investigación de tipo cuantitativo, transversal de alcance explicativo (Bernal, 2016), cuyo objetivo fue identificar la relación que existe entre la creatividad de un estudiante de Ingeniería en Computación y su rendimiento como programador, partiendo del supuesto de que la creatividad es incidente en el desarrollo de esta habilidad.

La investigación se realizó con estudiantes de dos grupos de Ingeniería en Computación de una universidad pública del estado de Jalisco (México). Participó un total de 64 alumnos, 34 de primer semestre y 30 de tercero, quienes cursaban las materias de Algoritmia y Estructura de datos respectivamente; para valorar la incidencia de la creatividad en su desempeño como programadores se tomó en cuenta el promedio obtenido en las asignaturas.

\subsection{La creatividad en el ámbito de estudiantes de Ingeniería en Computación}

Cuántas veces nos hemos maravillado por ser capaces de encontrar una respuesta inesperada a un problema que nos preocupaba, sin más conciencia que decir "hágase" y resultaba en su justa medida y proporción, y además de todo funcionaba (Corbálan, Martínez y Donolo, 2015). Lo mismo pudiera parecer el hecho de programar, ya que para autores como Newell (1969) la programación es una tarea comúnmente establecida como un ejercicio abierto en el que es difícil definir criterios analíticos y cuantitativos para juzgar la eficiencia y corrección de una solución.

Programar, por tanto, pareciera implicar el abordaje de problemas para encontrar una multitud de soluciones que dependerán de la capacidad de respuesta que tenga el individuo que realice dicha tarea. 
En este sentido, la literatura ofrece estudios que hablan de la creatividad, la programación y los estudiantes de ingeniería, entre otras temáticas que resulta interesante rescatar -en el sentido de conocer cómo se ha abordado esta materia en los últimos años.

Al respecto, Knobelsdorf y Romeike (2008) presentaron un estudio basado en testimonios de estudiantes de Ciencias Computacionales, quienes expresaban el motivo por el que elegían esa carrera. En los resultados identificaron que había alumnos creativos que preferían disponer de libertad y acrecentar su imaginación para crear productos nuevos conforme a un problema dado; los alumnos argumentaron que no es necesario sentarse a escuchar al profesor y tomar notas, pues esto es una limitante de tiempo y creatividad, por lo que prefieren trabajar de manera independiente. Por otra parte, identificaron a los estudiantes prácticos, quienes prefieren disponer de herramientas que les faciliten la programación que les lleve a encontrar soluciones lo más rápida y exactamente posible, sin dejar lugar a la experimentación, prefieren exprimir los conocimientos y habilidades del docente, sin ellos esforzarse demasiado.

Pérez-Poch, Olmedo y Salán (2015) realizaron un estudio sobre la influencia de la creatividad en el aprendizaje de la programación; para medirla, utilizaron el test de Torrance; los resultados indican que la creatividad no es influyente en el aprendizaje de todos los estudiantes de programación, sino sólo para aquellos que tienen una calificación más alta, es decir, los resultados sugieren que existe una correlación positiva entre alta creatividad y alto rendimiento como programadores, pero no una correlación en general en todos los estudiantes. Es decir, a rendimientos bajos y medios puede darse una alta o baja creatividad.

Por su parte, Deller (2013) asegura que en un grupo de estudiantes de programación la creatividad puede ser fomentada mediante una estructura de diálogo entre los alumnos, dándoles la oportunidad de plantear, complementar, rebatir y replantear las ideas de los demás, esto a partir de 7 pasos: inmersión, desenvolvimiento de divergencias de pensamiento, exploración de caminos complementarios, superación de límites mentales, expansión de ideas, análisis de malas ideas y desarrollo en conjunto de ideas y criterios.

Romeike (2007a) presentó un trabajo en el que describe un método de innovación para la enseñanza de las Ciencias Computacionales y argumentó que la creatividad raramente es aplicada por los estudiantes de Ciencias Computacionales debido a varias razones: los profesores los incitan a seguir caminos establecidos que parecen seguros y libres de riesgos, y que no dejan espacio para la creatividad; los estudiantes tienen diferentes metodologías de trabajo o diferentes software que les limita ser creativos, puesto que usan las herramientas para facilitar la programación -en este sentido, los profesores no fungen como motivadores de los alumnos, sino como guías para la realización de un proyecto estandarizado sin lugar para la variación. A este respecto, el autor estableció cuatro criterios para que las clases sean creativas:

1. Requerimientos sobre los temas: deben ser relevantes y enfocados a la creación de un producto o resolución de problemas.

2. Requerimientos sobre las actividades: deben ser abiertas a diferentes resultados, enfoques o métodos de solución, y al mismo tiempo aplicar los conceptos aprendidos.

3. Requerimientos del alumno: debe identificarse con la tarea y ser original.

4. Requerimientos del ambiente de enseñanza: debe existir apertura para experimentar, disponer de tiempo libre, retroalimentación entre estudiantes y la capacidad del maestro de trabajar como coach y no como evaluador.

Y concluye con que los métodos y herramientas de enseñanza, así como la capacidad y disponibilidad de los profesores, son clave para el desarrollo de la creatividad en los estudiantes (Romeike, 2007a).

Otra aportación es la de Shantanu (2017), quien habla de la necesidad de que los estudiantes desarrollen 
de manera natural la creatividad, guiados preferentemente por los docentes, de tal manera que se genere una dinámica de clases en la que los alumnos desarrollen el descubrimiento de conceptos o manipulen programas de forma gradual, incentivándolos a través de actividades que los obliguen a indagar, investigar y experimentar, lo que los llevaría a ampliar su capacidad de creación e invención.

Tang, Baer y Kaufman (2014) presentaron un estudio en el que implicaron el concepto de creatividad en las Ciencias Computacionales en instituciones de Estados Unidos y China, utilizando una escala tipo Likert en la que representaron cuatro dimensiones: la inteligencia efectiva, la extroversión, el pensamiento creativo y su capacidad de sociabilidad. A partir de su estudio concluyeron que existen diferentes tipos de creatividad y que cada una varía dependiendo el campo y el contexto de aplicación. El concepto de persona creativa está definido por la especialidad de ésta, y está sujeto a la interpretación del individuo del que viene la observación, la cultura y el contexto son influyentes en la importancia que se le dé al tema de creatividad; por ejemplo, para los chinos esta habilidad es más importante que para los estadounidenses.

Como se constata en los párrafos anteriores, la literatura expone la necesidad de vincular la creatividad a los procesos de enseñanza-aprendizaje de la programación; sin embargo, es evidente que aún hay mucho por investigar al respecto, ya que son pocos los estudios que entregan datos empíricos para corroborar esta relación.

\section{2 ¿Qué se entiende por creatividad?}

La creatividad no es sólo cosa de artistas, diseñadores industriales, empresarios, inventores, etc.; en realidad es capacidad de todos, ya que cualquier persona puede desarrollarla y aplicarla a partir de las situaciones que se vivan o los problemas que deba solucionar. Por lo difuso del concepto, éste encuentra significación en las teorías desde las que puede abordarse.

Runco y Sakamoto (1999, p. 62) afirman que "la creatividad se encuentra entre las más complejas conductas humanas. Parece estar influida por una amplia serie de experiencias evolutivas, sociales y educativas y se manifiesta de maneras diferentes en una diversidad de campos".

En las teorías psicológicas la más significativa es la de la creatividad incremental, en la que se considera el arraigo o la experiencia del individuo en su destreza y el desarrollo gradual de su trabajo anterior por medio de un proceso de pequeños y continuos saltos (Sánchez, García y Valdés, 2009).

Se entiende por persona creativa a aquel ser que genera ideas que sean relativamente nuevas, apropiadas y de alta calidad (Sternberg y Lubart, como se citó en Corbálan, Martínez y Donolo, 2015).

La creatividad es una de las formas donde el ser humano pone de manifiesto el resultado de su proceso de reflexión y sus propuestas a la solución de problemas; por lo tanto, el pensamiento creativo puede desarrollarse a través de la formación de estructuras de pensamiento, dando lugar inicialmente a la generación de ideas lógicas para posteriormente trascender a conceptos originales o no convencionales (Hernández, 2011).

La creatividad puede entenderse también como un cúmulo de factores que inciden en un proceso donde se tiene sensibilidad a los problemas y fluidez para generar ideas (Hernández, 2011).

Desde las Ciencias Computacionales la creatividad se caracteriza por la utilización de herramientas para crear otras en función de la necesidad de solucionar problemas eficazmente, aplicando la creación de programas (Saunders y Thagard, 2004).

Según Romeike (2007b), la oportunidad de ser creativo en las Ciencias Computacionales es un factor motivacional en los estudiantes. Por tanto, la creatividad está dirigida por tres elementos principales: la persona (estudiante), el ambiente y la motivación. La creatividad en la persona es intrínseca, y para desarrollarse se necesita una motivación (utilidad en resolución de problemas, reputación, identidad de 
grupo, etc.).

En este sentido, el concepto de creatividad y los estudios presentados se tomaron como referente para la realización de la investigación.

\section{Método}

A partir de la información proporcionada en los apartados anteriores puede observarse que el tema de la creatividad de los ingenieros en Computación -con énfasis en la incidencia que ésta tiene en las habilidades de programación- ha sido abordado escasamente, por lo que se decidió llevar a cabo una investigación que pudiera aportar algunos datos a este campo de conocimiento.

En este sentido, se planteó la siguiente pregunta de investigación: ¿Cuál es la relación que existe entre la creatividad de un estudiante de Ingeniería en Computación y su rendimiento como programador? Ante este supuesto se plantearon las siguientes hipótesis:

$\mathrm{H}_{0}$ : La creatividad no tiene relación con el rendimiento como programador de un estudiante de Ingeniería en Computación.

$\mathrm{H}_{1}$ : La creatividad tiene relación con el rendimiento como programador de un estudiante de Ingeniería en Computación.

Рara cumplir con los objetivos, contestar a la pregunta de investigación y comprobar las hipótesis señaladas, se diseñó una investigación cuantitativa transversal que se aplicó en una sola emisión (Hernández, Fernández y Baptista, 2014).

En el estudio participó un total de 65 estudiantes, 37 (57\%) alumnos que cursaban la asignatura de Algoritmia y 28 (43\%) la de Estructura de datos, la muestra se eligió a conveniencia del investigador (Hernández, Fernández, y Baptista, 2014) debido a las facilidades de acceso que se tenía con los grupos participantes y en los cuales era factible aplicar el instrumento a la totalidad de los alumnos inscritos en ambas asignaturas, además que por la naturaleza de éstas se relacionan directamente con habilidades de programación que los estudiantes deben desarrollar.

\subsection{Instrumento}

Para recabar los datos se utilizó el instrumento CREA (Inteligencia Creativa una medida cognitiva de la creatividad) de Corbalán, Martínez y Donolo (2015), el cual se sostiene en un modelo teórico dirigido a encontrar operaciones cognitivas no identificables en la producción creativa de los individuos.

Dicho instrumento fue validado en poblaciones españolas y de América Latina, por lo que se consideró que cuenta con la validez técnica y formas adecuadas para predecir y discriminar las medidas más clásicas de creatividad. Dicho test tiene las variantes A, B y C, mismas que se aplican a partir de la edad de los participantes, para el caso de esta investigación se utilizaron las variantes A y B dirigidas a adultos.

El instrumento se distribuyó entre los participantes y se les dieron las instrucciones sobre cómo manejarlo, ya que la prueba se realiza a través de imágenes y los alumnos debían escribir brevemente todas las preguntas que pudieran surgirles respecto a lo que se les presentaba. La indicación fue que escribieran el mayor número de preguntas posible en un lapso de cuatro minutos.

\subsection{Análisis de datos}

La imagen presentada a los estudiantes para el instrumento A consistía en un teléfono de los años cuarenta o cincuenta; en el caso B se presentó una escena un tanto grotesca en la que parecía que se estaban cortando las orejas a las personas que estaban ahí. 
Según las instrucciones del manual CREA, para validar la creatividad de los estudiantes habría primero que identificar que no quedaran lugares vacíos entre una línea y otra porque eso repercutiría en el cómputo final; en segundo lugar debían leerse todas las preguntas a fin de confirmar que se ajustaran a la instrucción dada. En principio todas las preguntas pueden considerarse correctas -excepto si existe alguna repetida, si está completamente descontextualizada o si son preguntas inacabadas como un ¿qué?, ¿cómo?, ¿cuándo?

Son consideradas como correctas aquellas que supongan la utilización de al menos un esquema cognitivo suscitado por la lámina, aunque ésta pueda parecer de un lenguaje elevado o su respuesta pudiera parecer poco común.

Finalmente, las preguntas que se hayan construido incluyendo dos o más cuestiones básicas se consideran con puntos dobles o triples, según sea el caso, el criterio es que la pregunta haga alusión a más de un esquema cognitivo.

Para realizar el cálculo se aplica la fórmula: PD = N - O - An + Ex

Donde: PD = Puntuación directa

N = Número correspondiente a la casilla donde se escribió la última pregunta

$\mathrm{O}=$ Corresponde al número de espacios vacíos u omisiones

An = Número de respuestas anuladas

Ex = Se anota el número de puntos extra por pregunta doble o triple

Una vez obtenida la puntuación directa se consultó en los baremos correspondientes para obtener la puntuación percentil del evaluado y proceder a su interpretación.

Revisión de los aspectos generales de las preguntas redactadas por los participantes:

1. Las preguntas de la imagen que hacen alusión a un teléfono en elaboración fueron más simples que las preguntas de aquella donde hay muestras en diferentes formas de orejas humanas.

2. Los renglones disponibles para elaborar preguntas eran 40 y el número máximo de interrogantes elaboradas sólo llegó a 20.

3. Hubo preguntas que se consideraron anuladas por referirse a los motivos de la aplicación de la prueba y el porqué de esas imágenes.

4. El promedio de preguntas redactadas en ambos ejercicios fue +-10 .

5. La imagen referente a orejas humanas despertó más el interés en los estudiantes, inclusive hubo quien manifestó que era una imagen agresiva.

6. En la imagen referente al teléfono las percepciones según las preguntas elaboradas redundaban en términos de los materiales, funcionalidad, antigüedad del aparato, entre otros; sin embargo, en la imagen de las orejas aparecieron preguntas más allá de la misma imagen; por ejemplo, en cuanto al sexo de las personas, la religión, incluso lo que sentían quienes participaban en la escena.

7. En todos los casos se utilizaron líneas continuas para contestar, es decir, no hubo espacios en blanco. 
8. Fueron muy pocas las preguntas con puntos extras, realmente sus elaboraciones fueron muy simples.

\section{Resultados}

A continuación, en la tabla I se observa la calificación de las asignaturas correspondientes, las puntuaciones obtenidas por cada estudiante en los ejercicios A y B, donde PD indica la puntuación directa y PC la relación con el percentil que el mismo manual del instrumento indica para la valoración, con este resultado se mide si la creatividad es Alta, Media o Baja. 
Tabla I. Tabla descriptiva de los resultados obtenidos en las pruebas de creatividad con los instrumentos A y B propios para medir la creatividad de adolescentes y adultos

\begin{tabular}{|c|c|c|c|c|c|c|}
\hline \multirow{3}{*}{$\begin{array}{c}\text { Calificación } \\
91\end{array}$} & \multicolumn{3}{|c|}{ Instrumento A } & \multicolumn{3}{|c|}{ Instrumento B } \\
\hline & \multirow{2}{*}{$\begin{array}{c}P D \\
-8\end{array}$} & \multicolumn{2}{|c|}{$P C$} & \multirow{2}{*}{$\begin{array}{c}\text { PD } \\
-12\end{array}$} & \multicolumn{2}{|c|}{ PC } \\
\hline & & 89 & Alta & & 82 & Alta \\
\hline 87 & -15 & 73 & Media & -10 & 89 & Alta \\
\hline 88 & -26 & 25 & Ваја & -28 & 27 & Media \\
\hline 87 & -24 & 43 & Media & -22 & 55 & Media \\
\hline 88 & -10 & 85 & Alta & -10 & 89 & Alta \\
\hline 92 & -27 & 19 & Baja & -22 & 55 & Media \\
\hline 95 & -10 & 85 & Alta & -2 & 99 & Alta \\
\hline 92 & -28 & 16 & Baja & -26 & 31 & Media \\
\hline 92 & -18 & 58 & Media & -22 & 55 & Media \\
\hline 68 & -24 & 43 & Media & -32 & 10 & Baja \\
\hline 64 & -16 & 69 & Media & -22 & 55 & Media \\
\hline 81 & -12 & 79 & Alta & -5 & 93 & Alta \\
\hline 87 & -28 & 16 & Ваја & -28 & 27 & Media \\
\hline 93 & -26 & 25 & Вaja & -28 & 27 & Media \\
\hline 71 & -30 & 05 & Вaja & -32 & 10 & Ваја \\
\hline 88 & -24 & 43 & Media & -24 & 38 & Media \\
\hline 81 & -18 & 58 & Media & -18 & 62 & Media \\
\hline 93 & -16 & 69 & Media & -12 & 82 & Alta \\
\hline 100 & -16 & 69 & Media & -24 & 38 & Media \\
\hline 85 & -29 & 08 & Вaja & -16 & 65 & Media \\
\hline 100 & -18 & 58 & Media & -29 & 14 & Ваја \\
\hline 87 & -20 & 47 & Media & -21 & 58 & Media \\
\hline 100 & -6 & 96 & Alta & -14 & 72 & Media \\
\hline 66 & -6 & 96 & Alta & -12 & 82 & Alta \\
\hline 93 & -24 & 43 & Media & -23 & 41 & Media \\
\hline 92 & -24 & 43 & Media & -28 & 27 & Media \\
\hline 85 & -14 & 75 & Alta & -14 & 72 & Media \\
\hline 100 & -32 & 01 & Вaja & -33 & 01 & Baja \\
\hline 93 & -20 & 52 & Media & -24 & 26 & Media \\
\hline 85 & -12 & 84 & Alta & -8 & 89 & Alta \\
\hline 98 & -12 & 84 & Alta & -18 & 57 & Media \\
\hline 83 & -24 & 22 & Baja & -20 & 55 & Media \\
\hline 87 & -20 & 52 & Media & -24 & 26 & Media \\
\hline 90 & -22 & 33 & Media & -24 & 26 & Media \\
\hline 91 & -26 & 14 & Вaja & -22 & 40 & Media \\
\hline 92 & -18 & 57 & Media & -20 & 55 & Media \\
\hline 89 & -20 & 52 & Media & -18 & 57 & Media \\
\hline 82 & -8 & 89 & Alta & -12 & 84 & Alta \\
\hline 86 & -10 & 86 & Alta & -14 & 73 & Media \\
\hline 70 & -18 & 57 & Media & -22 & 40 & Media \\
\hline 80 & -16 & 70 & Media & -20 & 55 & Media \\
\hline 83 & -4 & 94 & Alta & 2 & 99 & Alta \\
\hline 94 & -26 & 14 & Ваја & -26 & 16 & Ваја \\
\hline 73 & -14 & 73 & Media & -14 & 73 & Media \\
\hline 92 & -16 & 70 & Media & -22 & 40 & Media \\
\hline 100 & -20 & 52 & Media & -30 & 03 & Baja \\
\hline 82 & -24 & 22 & Вaja & -26 & 16 & Baja \\
\hline 88 & -26 & 11 & Baja & -26 & 16 & Ваја \\
\hline 93 & -22 & 33 & Media & -16 & 70 & Media \\
\hline 90 & -22 & 33 & Media & -22 & 40 & Media \\
\hline 92 & -20 & 52 & Media & -22 & 40 & Media \\
\hline 91 & -20 & 52 & Media & -20 & 55 & Media \\
\hline 95 & -12 & 84 & Alta & -18 & 57 & Media \\
\hline 83 & -20 & 52 & Media & -20 & 55 & Media \\
\hline 90 & -16 & 70 & Media & -28 & 05 & Вaja \\
\hline 91 & -12 & 84 & Alta & -18 & 57 & Media \\
\hline 87 & -26 & 14 & Вaja & -14 & 73 & Media \\
\hline 96 & -22 & 33 & Media & -16 & 70 & Media \\
\hline 85 & -16 & 70 & Media & -18 & 57 & Media \\
\hline 96 & -24 & 22 & Вaja & -20 & 55 & Media \\
\hline 80 & 4 & 99 & Alta & -4 & 94 & Alta \\
\hline 80 & -16 & 70 & Media & -10 & 86 & Alta \\
\hline 88 & -26 & 14 & Ваја & -26 & 16 & Ваја \\
\hline 86 & -4 & 94 & Alta & 2 & 99 & Alta \\
\hline
\end{tabular}


Como puede observarse en la tabla I, el valor PD en el $99 \%$ de los casos es negativo, esto debido a que la mayoría de estudiantes no alcanzó el número máximo de preguntas requeridas por el instrumento (40), de tal manera que al calcular la puntuación directa los valores se hacen negativos, recuérdese que el promedio de preguntas redactadas fue de +-10 .

Respecto al percentil que indica el instructivo para la interpretación en puntuación alta coinciden 29 aplicaciones, puntuación media 73 y puntuación baja 26, obsérvese en la tabla que hay alumnos que mantienen su nivel en ambos instrumentos y hay quienes no, en algunos casos decaen o se incrementa y hubo dos personas que no hicieron el segundo instrumento, también se nota que el promedio parece no tener relación con el nivel de creatividad que se presenta, ya que puede encontrarse que a promedios altos su nivel de creatividad es media o baja, o en algunos casos coincide que el promedio es alto y su nivel de creatividad también.

Para corroborar lo anterior y comprobar las hipótesis estadísticas planteadas:

- $\mathrm{H}_{0}$ : La creatividad no tiene relación con el rendimiento como programador de un estudiante de Ingeniería en Computación.

- $\mathrm{H}_{1}$ : La creatividad tiene relación con el rendimiento como programador de un estudiante de Ingeniería en Computación.

La prueba estadística $\mathrm{Chi}^{2}$ que permite medir la relación entre variables, (promedio y creatividad) se aplicó considerando los promedios y el dato de puntuación directa (PD), en este caso, se acepta la hipótesis nula y se rechaza la alternativa, puesto que para la aplicación del Instrumento A los valores obtenidos son: $p=.394$, significancia: 1.000 , y para el Instrumento $\mathrm{B} p=.830$ con significancia de 1.000 . Es decir, no hay relación entre la creatividad con el rendimiento como programador, puesto que el valor de significatividad es mayor a 0.05 en función del 95\% de confianza con el que se aplicó la prueba.

A partir del manual CREA la interpretación del nivel de creatividad se da en términos de:

- Quien obtiene un nivel alto es una persona con excelentes posibilidades para el desarrollo de tareas de innovación y producción creativa. Demuestra actitudes interrogativas ante el entorno. Tienen disponibilidad al cambio y para la búsqueda de información que les permita ser innovadores.

- Para las personas con nivel de creatividad medio, la producción creativa que presentan es moderada y no se destaca por sus capacidades de innovación o la búsqueda de soluciones alternativas a problemas. Su imaginación se ve un tanto limitada, sin embargo, se da la oportunidad de que en ocasiones aplique su habilidad creativa.

- Quienes tienen un nivel bajo, su producción creativa es limitada, no tienen un especial interés por cuestionar las características de su entorno, y se limitan a resolver problemas que no les exija un alto nivel cognitivo, son adaptables a las situaciones que les convienen y se rehúsan a la reflexión crítica.

\section{Discusión y conclusiones}

La investigación realizada permite afirmar que para el caso de los participantes de este estudio, la creatividad no es incidente en sus habilidades como programadores, puesto que su rendimiento académico no tiene relación con ésta, coincidiendo con la conclusión a la que llegaron Pérez-Poch, Olmedo y Salán (2015), quienes en sus resultados indican que la creatividad no influye en el aprendizaje de quienes estudian programación, sólo es notoria en algunos casos donde el promedio es alto y el nivel de creatividad también, pero no es un indicador estable. 
Los instrumentos que CREA propone para adolescentes y adultos incrementan el nivel de complejidad para la observación y abstracción de una prueba a otra, lo que da oportunidad a que los participantes puedan explayarse más en uno de los ejercicios que en el otro; sin embargo, en este caso los resultados fueron muy similares. Durante la aplicación se observó que los estudiantes esperaban algo más detallado o relacionado a una computadora, puesto que se hablaba de la creatividad de un programador, por lo que cambiarles el contexto y sacarlos de sus esquemas pudo ser el origen del poco número de preguntas redactadas en cada uno de los casos.

A partir del estudio se infiere que los ingenieros en Computación no se hacen conscientes de que ser creativos implica cualquier contexto o situación, y que esta habilidad les permite desarrollar mejores modelos abstractos para la solución de problemas o necesidades.

El tiempo otorgado para realizar el ejercicio (4 minutos), según las indicaciones de CREA, son suficientes para que se pueda elaborar un buen número de preguntas y en el mejor de los casos cuestionamientos que impliquen un alto grado cognitivo, desafortunadamente en este caso no fue así.

Tal como lo mencionan Romeike (2014a) y Shantanu (2017), se observó que la creatividad es una habilidad que debe promoverse por los docentes que imparten materias relacionadas a la programación, ya que presentar códigos a los estudiantes para que los copien y los compilen como una actividad para desarrollar la capacidad de programar no es suficiente, se requiere plantearles problemas que impliquen una solución eficaz y eficiente planteada en la lógica de un algoritmo y la codificación asociada a éste.

De tal manera que se considera necesario que los estudiantes sean creativos y que preferentemente el docente genere la dinámica de clase que permita el desarrollo de esta habilidad, donde ellos puedan descubrir soluciones, representen en lenguaje natural y formal sus propuestas de programas, manipulen lenguajes de programación y que a partir de las necesidades sepan elegir cuál es más conveniente para la solución del problema planteado.

El estudio también ayudó a identificar que, tal como lo expresaron Tang, Baer y Kaufman (2014), la creatividad se da o hace evidente a partir de los diferentes contextos en los que el estudiante está presente.

De igual manera pudo observarse que la fluidez para generar ideas no fue la habilidad más notoria de los participantes, por el contrario, su capacidad creativa se vio muy limitada quizá porque tuvieron que salir de su contexto y se les presentaron situaciones diferentes a las que esperaban, inferencia que coincide con lo explicado por Hernández (2011).

Por último, cabe comentar que aunque se explicó a los estudiantes lo que debían hacer, el motivo de la aplicación del instrumento y otros detalles más, ellos no se esforzaron por demostrar su capacidad creativa.

Es importante considerar la realización de más estudios en los que pueda identificarse la capacidad creativa de quienes se dedican a tareas de programación, ya que se parte de la premisa de que para programar se debe ser creativo, aunque en esta invetigación -así como en los estudios que se han tomado como referencia- al parecer no sucede así. Por tanto, la creatividad es una habilidad que requiere seguir analizándose para medir su impactos en el desempeño académico de los estudiantes.

\section{Referencias}

Bernal, C. (2016). Metodología de la Investigación. Bogotá: Pearson.

Corbálan, F. J., Martínez, F. y Donolo, D. S. (2015). CREA. Inteligencia Creativa. Una medida cognitiva de la creatividad (3a. ed.). Madrid: Tea.

Deller, J. (2013). Fostering the creative development of computer science students in programming and 
interaction design. Procedia Computer Science, 18, 1446-1455. doi:10.1016/j.procs.2013.05.312

Escobar, M. C. (2015). Mi taller digital. Una apuesta para el desarrollo de las habilidades tic en los estudiantes. Bits de Ciencia, 12, 44-47. Recuperado de https://www.dcc.uchile.cl/Bitsdeciencia12.pdf

Espeso, P. (10 de diciembre de 2015). Aprender a programar desarrolla la creatividad, la sociabilidad y mucho más. Educación 3.0. Recuperado de https://www.educaciontrespuntocero.com/opinion/aprenderprogramar-desarrolla-la-creatividad-la-sociabilidad-mucho-mas/30933.html

González, W., Estrada, V. y Martínez, M. (2016). Educrea, Educación Creativa. Recuperado de http://institucional.us.es/revistas/universitaria/27/art 1.pdf

Hernández, G. (2011). Pensamiento creativo: una propuesta para su desarrollo desde la programación de computadoras. Revista Unimar, 29(2), 115-122. Recuperado de http://editorial.umariana.edu.co/revistas/index.php/unimar/article/view/221

Hernández, R., Fernández, C. y Baptista, P. (2014). Metodología de la Investigación. México: McGrawHill.

Knobelsdorf, M. y Romeike, R. (2008). Creativity as a Pathway to Computer Science. ACM SICCSE Bulletin, 40(3), 286-290. doi:10.1145/1384271.1384347

Newell, A. (1969). Heuristic programming: illstructured programs. En J. Aronofsky (Ed.), Progress in operations research (Vol. 3, pp. 360-414). Nueva York: Wiley.

Pérez-Poch, A., Olmedo, N. y Salán, N. (julio, 2015). ¿Puede escuchar a los bits cantando? Estudio de la influencia de la creatividad en el aprendizaje de la programación. XXI Jornadas de la Enseñanza Universitaria de la Informática (pp. 209-215). Andorra la Vella: Universitat Oberta La Salle. Recuperado de https://upcommons.upc.edu/handle/2117/77109

Romeike, R. (2007a). Applying creativity in CS high school education-criteria, Teaching example and evaluation. En Proceedings of the Seventh Baltic Sea Conference on Computing Education Research (pp. 87-96). Koli, Finlandia. Recuperado de https://dl.acm.org/citation.cfm?id=2449323.2449333

Romeike, R. (2007b). Three drivers for creativity in computer science education. En Proceedings of the IFIPConference on Informatics, Mathematics and ICT: a golden triangle. Boston, EUA. Recuperado de http://ddi.cs.uni-potsdam.de/Forschung/Schriften/RomeikeIMICT2007.pdf

Runco, M. A. y Sakamoto, S. O. (1999). Experimental Studies of creativity. En R. J. Sternberg (Ed.), Handbook of creativity (pp. 62-92). Cambridge University Press.

Sánchez, P. A., García, A. y Valdés, Á. A. (2009). Validez y confiabilidad de un instrumento para medir la creatividad en adolescentes. Revista Iberoamericana de Educación, 50(6), 1-12. doi: 10.35362/rie5061939

Saunders, D. y Thagard, P. (2004). Creativity in computer science. En J. C. Kaufman y J. Baer (Eds.) Creativity across domains: faces of the muse (pp. 153-167). Mahwah, NJ: Lawrence Erlbaum Associates. Recuperado de http://cogsci.uwaterloo.ca/Articles/creativity.pdf

Shantanu, S. (2017). To teach computer science, creativity, programming. Recuperado de https://www.amplify.com/viewpoints/teach-computer-science-start-creativity (enlace no activo)

Tang, C., Baer, J. y Kaufman, J. C. (2014). Implicit theories of creativity in computer science in the United States and China. Journal of Creative Behavior, 4(2), 137-156. doi:10.1002/jocb.61 
Yates, R. (1999). Educación, Computación y Tecnología: Visión en la Antesala de un Nuevo Milenio. En Taller Internacional de Software Educativo (TISE). Santiago, Chile. Recuperado de

http://www.c5.cl/ieinvestiqa/actas/tise99/html/fcharlas.html 